\title{
Elimination of Unintended Reflection Waves from a Test Site for Antenna Calibration using the MUSIC Algorithm
}

\author{
Non-member Harima Katsushige (Communications Research Laboratory, M.P.T.)
}

The antenna used for EMI measurements is calibrated at test sites, such as open area test sites or in anechoic chambers. The unintended reflection waves from the test sites cause uncertainty in the measurements. In this paper, the MUSIC algorithm was used to eliminate the unintended reflection waves from the test sites, and the antenna gain was determined by using the three-antenna method.

Keywords: antenna calibration, MUSIC algorithm, three-antenna method, uninteded reflection wave

\section{Introduction}

The antenna used for EMI measurements is calibrated at test sites, such as open area test sites or in anechoic chambers. The unintended reflection waves from the test sites cause uncertainty in the measurements. The time-domain analysis technique based on the fast Fourier transform (FFT) is the method often used to estimate and remove these reflection waves. However, the time-response resolution is restricted by the frequency bandwidth. On the other hand, it is well known that the MUSIC algorithm (Multiple Signal Classification) ${ }^{(1)}$ and ESPRIT algorithm (Estimation of Signal Parameters via Rotational Invariance Techniques) ${ }^{(2)}$, the supperresolution technique for the estimation of multiple arrival waves using the antenna array, can obtain higher resolution than FFT.

In this paper, the MUSIC algorithm was used to eliminate the unintended reflection waves from the test sites, and the antenna gain was determined by using the three-antenna method.

2. The Three-antenna Method in Free-space

The antenna insertion loss is defined as the ratio of the received power by the receiving antenna to the input power at the transmitting antenna. If the two antennas are arranged in a free-space as shown in Fig. 1, the relationship between the antenna insertion loss $\left(P L_{t r}\right)$ and the antenna gains can be expressed by the Fris transmission formula as;

$$
G_{t} G_{r}=P_{r} / P_{t}(4 \pi d / \lambda)^{2}=P L_{t r}(4 \pi d / \lambda)^{2} \ldots
$$

where $G_{t}$ is the gain of the transmitting antenna, $G_{r}$ is the gain of the receiving antenna, $P_{t}$ is the input power to the transmitting antenna, $P_{r}$ is the power received by the receiving antenna, $d$ is the distance between the two antennas, and $\lambda$ is the wavelength.

We can determine the free-space antenna gain for each by solving three similar equations to (1) obtained three pairs of antenna. For example, the gain of one of three antennas is given by

$$
G_{1}=\frac{4 \pi d}{\lambda} \sqrt{\frac{P L_{12} P L_{13}}{P L_{23}}} \frac{\left|\left(1-\Gamma_{1} \Gamma_{g}\right)\left(1-\Gamma_{1} \Gamma_{l}\right)\right|}{\left(1-\left|\Gamma_{1}\right|^{2}\right)\left|1-\Gamma_{g} \Gamma_{l}\right|}
$$

where $\Gamma_{1}, \Gamma_{g}$, and $\Gamma_{l}$ are the reflection coefficients of the connecting points of the antenna and coaxial cable.

\section{The MUSIC Algorithm}

Let us assume that $D$ incident waves arrive at the receiving antenna in the measurement system, as shown in Fig. 1. The received complex value as a sum of the arrival waves at the frequency $f$ is given by

$$
r_{f}=\sum_{k=1}^{D} s_{k} e^{-j 2 \pi f t_{k}}+n_{f} \ldots \ldots \ldots \ldots \ldots \ldots \ldots
$$

where $s_{k}$ is the complex signal strength of the $k$ th arrival wave, $t_{k}$ is the delay time of the $k$ th arrival wave, and $n_{f}$ denotes additive white Gaussian noise with zero mean and variance $\sigma^{2}$. If we rewrite (3) in vector notation for $L$ frequency samples $\left(f_{i}=f_{1}\right.$ to $\left.f_{L}\right)$, we obtain

$$
r=A s+n
$$

where

$$
\begin{aligned}
r & =\left[\begin{array}{llll}
r_{1} & r_{2} & \ldots & r_{L}
\end{array}\right]^{T} \\
A & =\left[\begin{array}{llll}
a\left(t_{1}\right) & a\left(t_{2}\right) & \ldots & a\left(t_{D}\right)
\end{array}\right] \\
s & =\left[\begin{array}{llll}
s_{1} & s_{2} & \ldots & s_{D}
\end{array}\right]^{T} \\
n & =\left[\begin{array}{lllll}
n_{1} & n_{2} & \ldots & n_{L}
\end{array}\right]^{T} \\
a\left(t_{k}\right) & =\left[\begin{array}{lllll}
e^{-j 2 \pi f_{1} t_{k}} & e^{-j 2 \pi f_{2} t_{k}} & \ldots & e^{-j 2 \pi f_{L} t_{k}}
\end{array}\right]^{T} .
\end{aligned}
$$

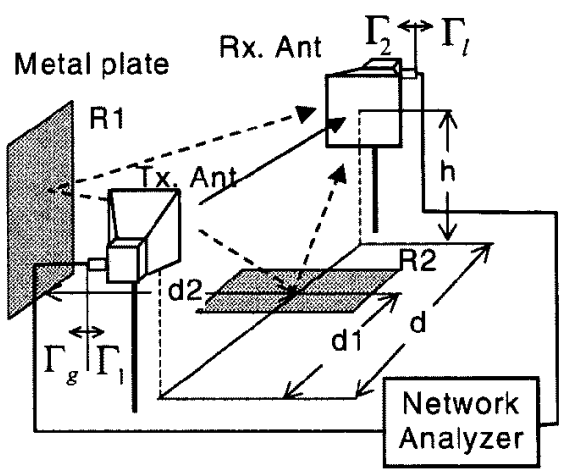

Fig. 1. Experimental set-up. 
Here, ${ }^{T}$ denotes the transpose. If the data obtained from (4) are incoherent, the MUSIC algorithm can be used to estimate the delay time $t_{k}$ of the reflection waves by the correlation matrix of the received data. In the MUSIC algorithm, the correlation matrix is defined as

$$
R=\left\langle r \cdot r^{H}\right\rangle \ldots \ldots \ldots \ldots \ldots \ldots \ldots \ldots \ldots \ldots \ldots \ldots
$$

where ${ }^{H}$ denotes the complex conjugate transpose, and $\langle\cdot\rangle$ denotes the ensemble average. The eigenvectors of the correlation matrix are calculated in order to estimate the number of the arrival waves. We have the eigenvalues $\left\{\lambda_{1} \geq \lambda_{2} \geq \cdots \geq \lambda_{D} \geq \lambda_{D+1} \cong \lambda_{D+2} \cong\right.$ $\left.\cdots \cong \lambda_{L} \cong \sigma^{2}\right\}$ corresponding the signal $\left(\lambda_{1}\right.$ to $\left.\lambda_{D}\right)$ and the noise $\left(\lambda_{D+1}\right.$ to $\left.\lambda_{L}\right)$, and similarly the eigenvectors $\left\{e_{1}, e_{2}, \cdots, e_{L}\right\}$ corresponding the signal and the noise $\left(\left\{e_{D+1}, e_{D+2}, \cdots, e_{L}\right\}=E_{N}\right)$. The delay time of each reflection wave can be estimated by searching for the peak position of the following function defined by using the eigenvectors $E_{N}$ and the mode vector $a\left(t_{k}\right)$ in $(4)^{(3)}$.

From (4), assuming the noise with zero mean, the vector $S$ is given by ${ }^{(4)}$

$$
s=\left(A^{H} A\right)^{-1} A^{H} \text { r. . . . . . . . . . . . . . . }
$$

The unintended reflection waves can be eliminated by putting " 0 " to the vector $s$ corresponding to the reflection waves. Thus, the received data in free-space without the reflection waves can be obtained by

$$
P L_{t r}=|r|=\left|\mathbf{A}\left[\begin{array}{lll}
s_{1} & 0 & 0
\end{array}\right]^{\mathrm{T}}\right| . \ldots \ldots \ldots \ldots .
$$

\section{Experimental Results}

The experimental set-up we used to determine the antenna gain by applying the MUSIC algorithm is shown in Fig. 1. A transmitting and a receiving antenna were connected to a network analyzer and arranged in an anechoic chamber at same height $h(=0.95 \mathrm{~m})$ and a distance $d(=5 \mathrm{~m})$. Two metal boards $(R 1, R 2)$ as the reflection points were placed at each position $\left(d_{1}\right.$ $=2.5 \mathrm{~m}, d_{2}=2.2 \mathrm{~m}$ ) in order to estimate the performance of the MUSIC algorithm. Three double ridge horn antennas were used for the three-antenna method. We measured the received data ( $S_{21}$ parameter) at 1601 samples from 1 to $3 \mathrm{GHz}$ by using the network analyzer for the three pairs of antennas. The received data that was obtained are coherent. Thus, we preprocessed the data in order to apply the MUSIC algorithm using the modified spatial smoothing technique ${ }^{(5)}$ to destroy the signal coherence.

Figure 2 shows the estimation result of the delay time of the arrival waves to the receiving antenna using the MUSIC and inverse FFT (IFFT) algorithms. In this calculation, the data were used for $401(=L)$ samples from 2 to $2.5 \mathrm{GHz}$. The three peaks corresponding to a direct wave $\left(t_{1}=18.7 \mathrm{~ns}\right)$ and two reflection waves ( $t_{2}=20.0 \mathrm{~ns}, t_{3}=24.1 \mathrm{~ns}$ ) from the metal boards can be detected by applying the MUSIC algorithm. The results of the IFFT cannot identify the arrival waves.

Figure 3 shows the antenna gain in free-space determined by using the three-antenna method. The unintended reflection waves due to the metal boards estimated by applying the MUSIC algorithm were eliminated by $(7)$ in order to obtain the received data $\left(P L_{t r}\right)$,

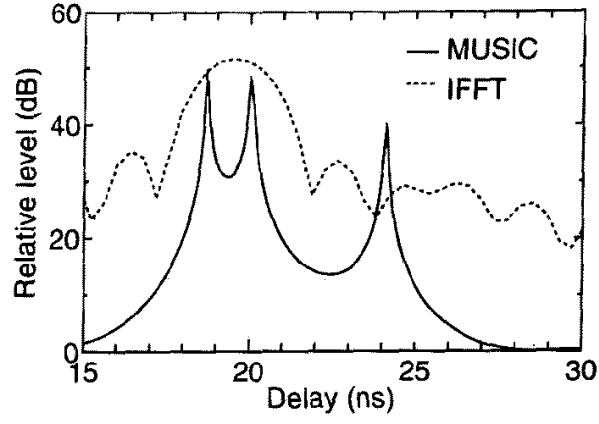

Fig. 2. Estimated results of the arrival waves using MUSIC and IFFT.

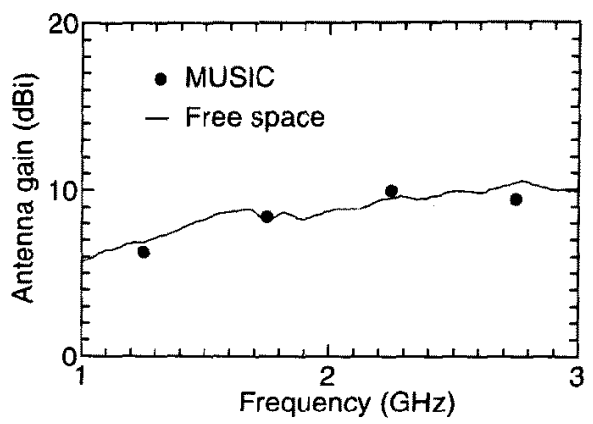

Fig. 3. Antenna gain determined by the three antenna method. The reflection waves are eliminated by applying the MUSIC and removing all reflection boards.

and then the antenna gains were determined by (2). To confirm these results, all the reflection boards were removed from the anechoic chamber, and then the antenna gains determined were compared in the same figure.

\section{Conclusion}

We have eliminated the unintended reflection waves of the test site using the MUSIC algorithm, and determined the free-space antenna gain by using the threeantenna method. The experimental results showed that the MUSIC algorithm was better than the IFFT at estimating and eliminating of the unintended reflection waves from the test site.

(Manuscript received February 9, 2000)

\section{References}

(1) R. O. Schmidt, "Multiple emitter location and signal parameter estimation," IEEE Trans., AP-34, 3, pp. 276-280 (1986)

(2) R. Roy and T. Kailath, "ESPRIT-Estimation of signal parameters via rotational invariance techniques," IEEE Trans., ASSP-37, 7, pp. 984-995 (1989).

(3) H. Yamada, M. Ohmiya, Y. Ogawa and K. Itoh, IEEE Trans. "Super-resolution techniques for time-domain measurements with a network analyzer," AP-39, 2, pp. 177-183 (1991).

(4) H. Yamada, Y. Ogawa and K. Itoh, "Superresolution techniques for antenna gain measurements with a net-work analyzer," IEICE Tech. Report, AP91-106, pp. 31-38 (1991).

(5) R. T. Williams, S. Prasad, A. K. Mahalanabis and L. H. Sibul, "An improved spatial smoothing technique for bearing estimation in a multipath environment," IEEE Trans., ASSP-36, 4, pp. $425-432$ (1988). 